\section{Desigualdades contextuais e individuais da prevalência de dor dentária em adultos e idosos no Brasil}

\author{
Contextual and individual inequalities in dental \\ pain prevalence among Brazilian adults and elders
}

\author{
1 Programa de Pós- \\ graduação em Saúde \\ Coletiva, Universidade \\ Federal de Santa Catarina, \\ Florianópolis, Brasil. \\ 2 Secretaria de Vigilância em \\ Saúde, Ministério da Saúde, \\ Brasília, Brasil. \\ 3 Programa de Pós-graduação \\ em Epidemiologia, \\ Universidade Federal do Rio \\ Grande do Sul, Porto Alegre, \\ Brasil. \\ 4 Escola de Enfermagem, \\ Universidade Federal de \\ Minas Gerais, Belo Horizonte, \\ Brasil. \\ 5 Faculdade de Saúde \\ Pública, Universidade de São \\ Paulo, São Paulo, Brasil. \\ Correspondência \\ M. A. Peres \\ Programa de Pós-graduação \\ em Saúde Coletiva, Centro \\ de Ciências da Saúde, \\ Universidade Federal de Santa \\ Catarina. \\ Campus Universitário \\ Florianópolis, SC 88037-325, \\ Brasil. \\ mperes@ccs.ufsc.br
}

\section{Abstract}

This study aimed to assess the prevalence of dental pain among adults and older people living in Brazil's State capitals. Information was gathered from the Telephone Survey Surveillance System for Risk and Protective Factors for Chronic Diseases (VIGITEL) in 2009 ( $n=54,367)$. Dental pain was the outcome. Geographic region, age, gender, race, schooling, private health coverage, smoking, and soft drink consumption were the explanatory variables. Multilevel Poisson regression models were performed. Prevalence of dental pain was 15.2\%; Macapá and São Luís had prevalence rates greater than 20\%; all capitals in the South and Southeast, plus Cuiabá, Campo Grande, Maceió, Recife, and Natal had prevalence rates less than 15\%. Factors associated with increased prevalence of dental pain were the North and Northeast regions, female gender, black/brown skin color, lack of private health insurance, smoking, and soft drink consumption. Dental pain is a public health problem that should be monitored by health surveillance systems.

Toothache; Health Surveys; Dental Health Surveys; Oral Health

\author{
Marco A. Peres 1 \\ Betine Pinto Moehlecke Iser 2,3 \\ Karen Glazer Peres 1 \\ Deborah Carvalho Malta 2,4 \\ José Leopoldo Ferreira Antunes 5
}

\section{Introdução}

A dor dentária é um dos sintomas mais comuns das doenças e agravos bucais, é fortemente associada com a percepção de necessidade de cuidados odontológicos e exerce impacto na qualidade de vida 1 . Em adultos, o impacto da dor dentária inclui a perda de produtividade no trabalho, mudanças nos hábitos alimentares com restrição a certos alimentos ${ }^{2}$ e a necessidade de medicação e procura por serviços de saúde 1,3,4.

A dor dentária é um dos principais motivos para a procura de serviços de saúde e a busca por atendimento odontológico. Estudos mostram que cerca de $40 \%$ das pessoas que sentem dor procuram atendimento médico ou odontológico 1,5. No levantamento epidemiológico nacional de saúde bucal de 2003 6, a experiência de dor dentária foi o motivo da visita ao dentista para $46 \%$ dos adultos. Em estudo conduzido com a população de 14 a 49 anos no Sul do Brasil, percebeu-se que indivíduos do sexo masculino, de 35 a 49 anos, com baixa renda e escolaridade relataram ter consultado com ao dentista somente quando sentiam dor de dente 7 .

Fatores como o número de dentes perdidos por cárie 8 e o padrão de consultas com dentista 9 são associados à prevalência de dor dentária. Além disso, a prevalência de dor de dente tem sido relatada como sendo mais elevada em meninas e mulheres 2,5 , nas pessoas de menor esco- 
laridade e renda 10,11,12, em adultos jovens 5 e em indivíduos com menor acesso a cuidados básicos de saúde 13. O lugar de residência (urbano ou rural), o grau de desenvolvimento social (maior ou menor Índice de Desenvolvimento Humano - IDH) e a frequência de escovação dentária também interferem na prevalência de dor dentária 11,14, assim como o tabagismo, o consumo de álcool, hábitos alimentares não saudáveis e a presença de cáries dentárias 2,15,16. Embora a diferença de prevalência da dor dentária entre raças não seja muito explorada, esta parece ser maior entre os negros 4,17, nos quais o agravo apresenta maior impacto, como por exemplo, uma maior redução das atividades cotidianas e maior motivação pela procura de resolução da dor ${ }^{9}$. Por ser um sintoma subjetivo, fatores cognitivos como o conhecimento, crenças e expectativas, bem como aspectos culturais, têm sido associados a diferenças de percepção da dor 13 .

Apesar de ser facilmente obtida por meio de questionário face a face ou entrevistas telefônicas, de ser um bom indicador das condições de saúde bucal e propiciar uma avaliação indireta dos serviços odontológicos, apenas no final da década de 2000 sua investigação foi incluída em inquéritos de saúde de base populacional como o Sistema de Vigilância de Fatores de Risco e Proteção para Doenças Crônicas por Inquérito Telefônico (VIGITEL) e a Pesquisa Nacional de Saúde do Escolar (PeNSE) 18. Além disso, a coleta de informações sobre dor dentária está no elenco de questões a serem formuladas quando da Pesquisa Nacional de Saúde, planejada para ser realizada pelo Ministério da Saúde e Instituto Brasileiro de Geografia e Estatística (IBGE) em 201319.

O VIGITEL oferece uma excelente oportunidade de se conhecer a distribuição da prevalência de dor dentária em adultos e idosos das capitais brasileiras, e sua associação com indicadores de desenvolvimento social das regiões do país e características demográficas, socioeconômicas e comportamentais dos indivíduos. Permitirá também, por sua característica contínua, o monitoramento do agravo ao longo do tempo. Estudos com esta abrangência e características dirigidos a esta temática são inéditos no Brasil.

Este estudo tem como objetivo conhecer a prevalência de dor dentária e fatores contextuais e individuais associados em adultos e idosos residentes nas capitais do Brasil, identificando as desigualdades na distribuição desses fatores. Adicionalmente, estimou-se a associação entre indicador de desenvolvimento social da região geográfica de residência do participante e a dor dentária após o ajuste por variáveis individuais.

\section{Métodos}

Foram avaliados os dados provenientes do módulo de saúde bucal incluído no VIGITEL, no ano de 2009 , realizado entre 12 de janeiro a 22 de dezembro. O VIGITEL vem sendo realizado anualmente pelo Ministério da Saúde desde 2006, nas 26 capitais de todos os estados e Distrito Federal. O sistema utiliza uma amostra da população de 18 anos ou mais residente nas capitais do Brasil, em domicílios servidos por pelo menos uma linha de telefone fixo.

Foi obtida uma amostra probabilística em três estágios. A partir do recebimento dos cadastros das principais companhias telefônicas que cobrem cada cidade, a primeira etapa da amostragem consistiu no sorteio sistemático de 5 mil linhas telefônicas, as quais foram divididas em réplicas ou subamostras de 200 linhas cada, reproduzindo a mesma proporção de linhas por região da cidade ou prefixo telefônico. A divisão da amostra integral em réplicas foi realizada principalmente pela dificuldade em estimar previamente a proporção das linhas do cadastro elegíveis para o sistema e, portanto, o total de linhas a ser sorteado para se chegar ao mínimo amostral de 2 mil entrevistas por cidade. A segunda etapa da amostragem, realizada simultaneamente à realização das entrevistas, incluiu a identificação de linhas efetivamente residenciais e ativas, as quais foram consideradas elegíveis para o sistema. Nessa etapa foram excluídas as linhas empresariais, fora de serviço ou inexistentes, e os números que não responderam a seis ligações realizadas em horários e dias diferentes. A partir da identificação das linhas elegíveis, seguiu-se para a 3a etapa da amostragem, que consistiu no sorteio do indivíduo a ser entrevistado, dentre todos os adultos com 18 anos ou mais residentes no domicílio 20,21.

Considerou-se uma amostra mínima de 2000 entrevistas por cidade, para que se pudesse estimar com nível de $95 \%$ de confiança e erro máximo de cerca de dois pontos percentuais a frequência de qualquer fator de risco na população adulta e idosa, totalizando 54 mil entrevistas em todo o território nacional. As estimativas produzidas foram ajustadas à população adulta brasileira, a partir do uso de um peso pós-estratificação, calculado com base em 36 categorias de análise por sexo, faixa etária e escolaridade, segundo os dados do último censo demográfico (2000). A Figura 1 sumariza o processo de seleção da amostra.

O aplicativo utilizado na coleta de dados do VIGITEL permitiu o sorteio automático da pessoa a ser entrevistada, o salto automático de questões não aplicáveis em face de respostas anteriores, a 
Processo de seleção da amostra do Sistema de Vigilância de Fatores de Risco e Proteção para Doenças Crônicas por Inquérito Telefônico (VIGITEL), Brasil, 2009.

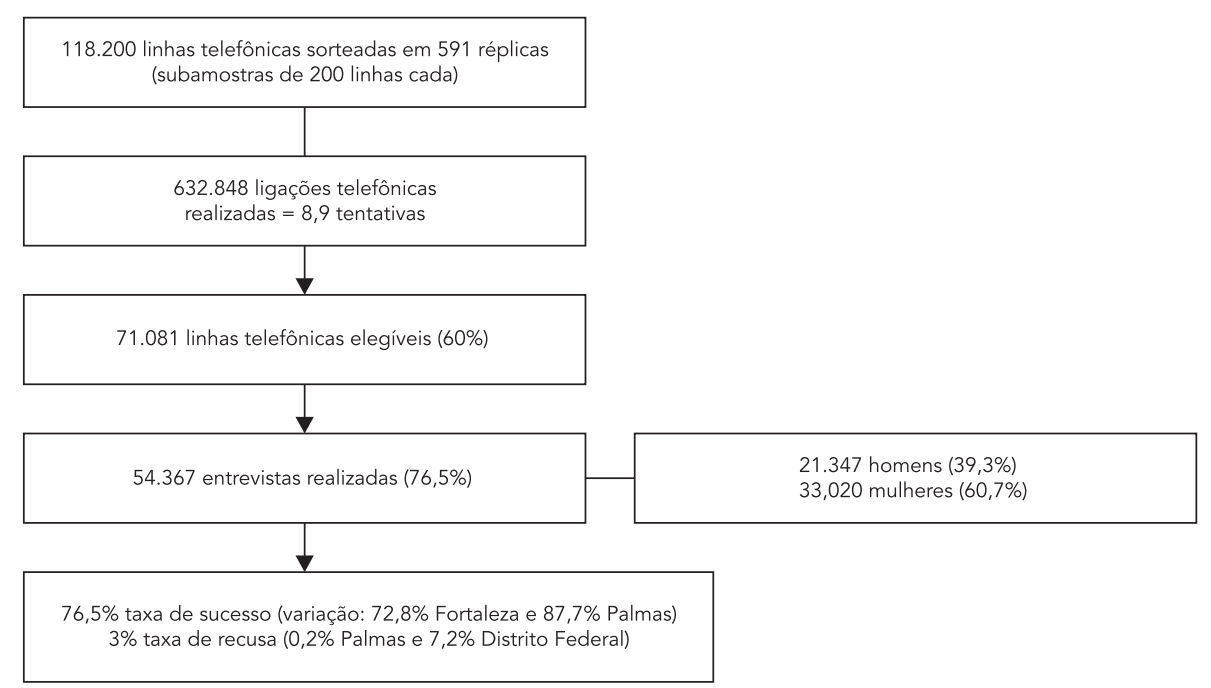

crítica imediata de respostas não válidas e a cronometragem da entrevista, além de propiciar a alimentação direta e contínua no banco de dados do sistema. A característica de automatização do aplicativo permitiu minimizar erros decorrentes de digitação incorreta, produzir relatórios parciais e fornecer subsídios para o controle gerencial de agendamentos e entrevistas. Além disso, todas as entrevistas foram gravadas e cerca de $10 \%$ auditadas pelos supervisores, como forma de controle de qualidade dos dados.

Em 2009, além das 94 questões específicas do VIGITEL, foram incluídas questões relacionadas à saúde bucal, sendo uma delas relativa à dor. Os entrevistados foram questionados sobre a experiência de dor dentária da seguinte forma: "Nos últimos 30 dias, o(a) Sr(a) teve algum problema como dor de dente ou inflamação da gengiva?". Neste estudo utilizamos como variáveis independentes a macrorregião geográfica do país, faixa etária, sexo, raça/cor da pele, escolaridade, posse de convênio médico, frequência de consumo de refrigerantes e tabagismo.

A análise geográfica entre as regiões Norte/ Nordeste e Sul/Sudeste/Centro-Oeste foi construída a partir da observação de que o máximo IDH das capitais do primeiro grupo foi 0,806 , enquanto o mínimo IDH das capitais do segundo grupo foi $0,814^{22}$. A partir da informação da ida- de em anos, foram construídas três faixas etárias: 35 anos ou menos; 36 a 59 anos; 60 anos ou mais. A cor da pele foi categorizada em branca, parda, preta ou outra. A escolaridade, a partir da informação de anos completos de estudo, incluiu as seguintes categorias de análise: 12 anos ou mais; 9 a 11; 5 a 8 anos e até 4 anos. Quanto ao convênio médico ou plano de saúde, o indivíduo foi classificado em: segurado, não segurado, não informado. Nas questões comportamentais, considerou-se a frequência de consumo de refrigerantes como menos do que três vezes na semana e três ou mais vezes na semana. Quanto ao tabagismo, o entrevistado foi classificado como não fumante ou fumante atual.

A avaliação dos fatores associados à prevalência de dor dentária utilizou análise de regressão de Poisson multinível, usando o modelo de efeitos fixos e intercepto randômico 23 , considerando dois níveis de organização dos dados: os entrevistados e a macrorregião do país. Foram estimadas para cada variável a razão de prevalências e o respectivo intervalo de $95 \%$ de confiança. A estratégia de análise seguiu uma estrutura conceitual de determinação da dor dentária. A região do país foi considerada a variável contextual; gênero, idade e cor da pele representaram as variáveis demográficas; escolaridade e possuir plano de saúde foram consideradas como 
proxy de condições socioeconômicas e, por último, consumo de refrigerantes (um indicador da frequência de consumo de açúcar) e tabagismo (marcador de risco para doença periodontal) foram as variáveis mais proximais ao desfecho. Todas as razões de prevalência foram ajustadas pelas variáveis situadas no mesmo nível de determinação e pelas variáveis situadas nos níveis mais distais 24. Posteriormente, a variável contextual - macrorregião - foi ajustada pelas variáveis que representavam as características individuais, para avaliar o quanto da associação no nível contextual podia ser explicado pelos fatores individuais identificados. Todas as análises foram realizadas no programa Stata, versão 9.2 (Stata Corp., College Station, Estados Unidos).

O consentimento livre e esclarecido foi substituído pelo consentimento verbal no momento do contato telefônico com o entrevistado. O estudo foi aprovado pela Comissão Nacional de Ética em Pesquisa em Seres Humanos, do Ministério da Saúde, sob parecer nº. 749/2006.

\section{Resultados}

Em todo o país, foram realizadas 54.367 entrevistas, 21.347 com homens e 33.020 com mulheres. A taxa de participação foi de 76,5\% (mínima de $72,8 \%$ em Fortaleza e máxima de $87,7 \%$ em Palmas). As recusas ocorreram no contato inicial (na residência) ou após o sorteio da pessoa a ser entrevistada. A duração média de cada entrevista foi de 11 minutos, variando de 9,5 minutos no Distrito Federal a 12,3 minutos em Macapá).

A prevalência de dor dentária foi de $15,2 \%$ e apresentou variação nas capitais. Prevalências maiores do que $20 \%$ foram identificadas em Macapá e São Luís, enquanto prevalências menores do que $15 \%$ foram encontradas em todas as capitais do Sul e Sudeste, em Cuiabá e Campo Grande no Centro-oeste e em Maceió, Recife e Natal na Região Nordeste (Figura 2).

A Tabela 1 apresenta a prevalência de dor dentária segundo as macrorregiões do país e as características individuais da amostra estudada.

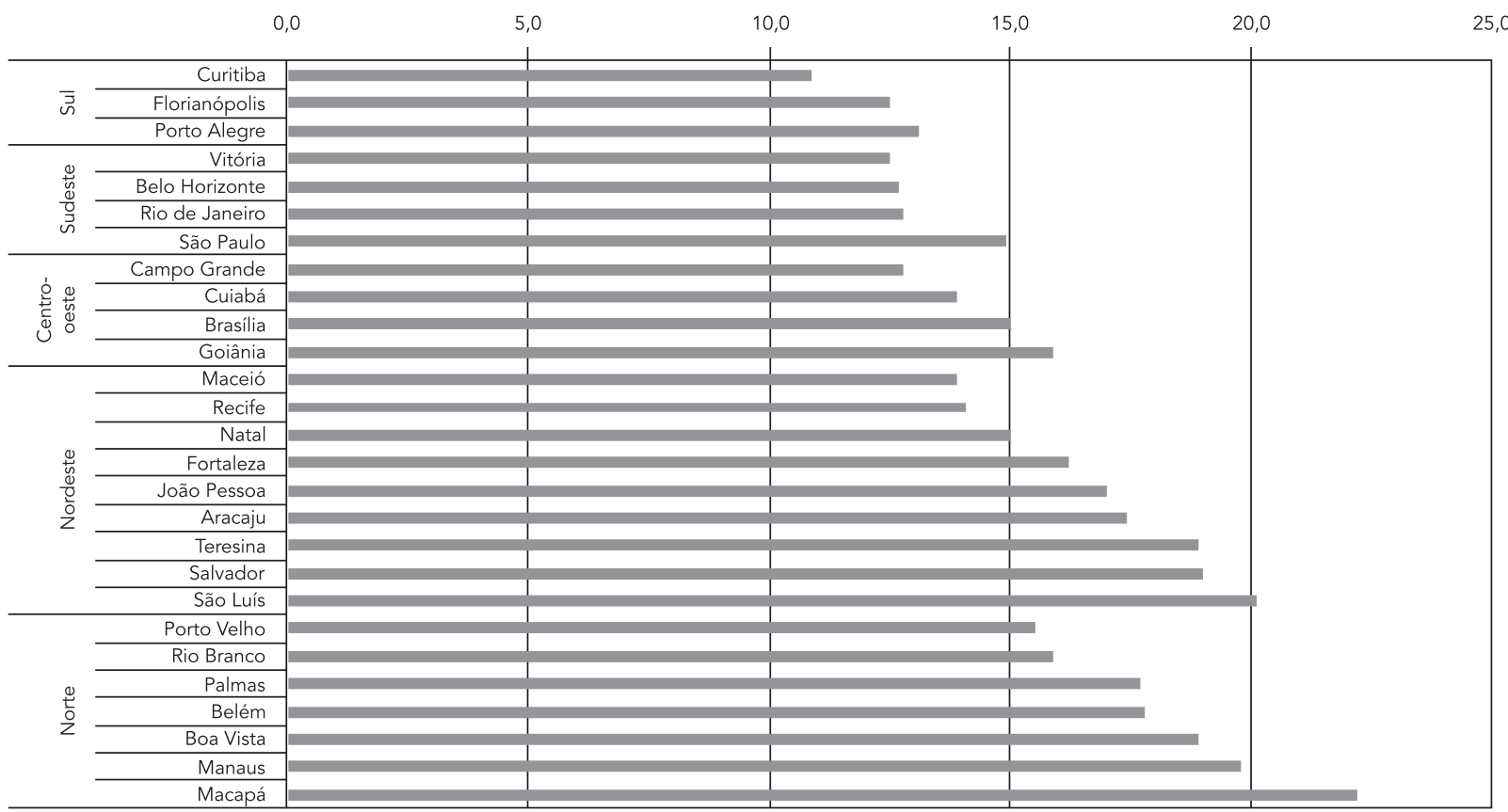


Tabela 1

Prevalência de dor dentária em adultos e idosos segundo região geográfica, características demográficas, socioeconômicas e comportamentais. Sistema de Vigilância de Fatores de Risco e Proteção para Doenças Crônicas por Inquérito Telefônico (VIGITEL), Brasil, 2009.

\begin{tabular}{|c|c|c|}
\hline Variáveis/Categorias & Frequência (\%) & Dor dentária (\%) \\
\hline \multicolumn{3}{|l|}{ 1ㅇ nível: contexto } \\
\hline \multicolumn{3}{|l|}{ Região geográfica } \\
\hline Sul, Sudeste e Centro-oeste & 63,3 & 13,9 \\
\hline Norte e Nordeste & 36,7 & 17,4 \\
\hline \multicolumn{3}{|l|}{ 2o nível: indivíduos } \\
\hline \multicolumn{3}{|l|}{ Características demográficas } \\
\hline \multicolumn{3}{|l|}{ Faixa etária (anos) } \\
\hline $18-35$ & 48,9 & 18,8 \\
\hline $36-59$ & 38,6 & 12,9 \\
\hline$\geq 60$ & 12,5 & 8,0 \\
\hline \multicolumn{3}{|l|}{ Gênero } \\
\hline Feminino & 53,9 & 17,2 \\
\hline Masculino & 46,1 & 12,8 \\
\hline \multicolumn{3}{|l|}{ Raça/Cor da pele } \\
\hline Brancos & 36,5 & 11,9 \\
\hline Pardos & 55,0 & 16,4 \\
\hline Pretos & 7,9 & 21,8 \\
\hline Outros & 0,6 & 13,4 \\
\hline \multicolumn{3}{|l|}{ Posição socioeconômica } \\
\hline \multicolumn{3}{|l|}{ Instrução (anos de estudo) } \\
\hline$\geq 12$ & 32,3 & 15,5 \\
\hline $9-11$ & 39,7 & 15,7 \\
\hline $5-8$ & 15,1 & 13,6 \\
\hline$\leq 4$ & 12,4 & 14,5 \\
\hline Não informado & 0,5 & 13,6 \\
\hline \multicolumn{3}{|l|}{ Plano privado de saúde } \\
\hline Não possui & 57,4 & 18,7 \\
\hline Possui & 42,5 & 10,4 \\
\hline Não informado & 0,1 & 1,8 \\
\hline \multicolumn{3}{|l|}{ Comportamento } \\
\hline \multicolumn{3}{|c|}{ Consumo de refrigerantes (vezes por semana) } \\
\hline$<3$ & 57,0 & 12,9 \\
\hline$\geq 3$ & 43,0 & 18,2 \\
\hline \multicolumn{3}{|l|}{ Hábito de fumar } \\
\hline Não fumantes & 84,5 & 14,1 \\
\hline Fumantes & 15,5 & 21,0 \\
\hline Total & 100,0 & 15,2 \\
\hline
\end{tabular}

A prevalência de dor dentária foi maior nas capitais das regiões Norte e Nordeste, nas mulheres, nos mais jovens, nos de cor de pele preta, nos fumantes e nos que não possuem planos de saúde.

Residentes nas regiões Norte e Nordeste apresentaram prevalência de dor dentária $25 \%$ mais elevada do que entre os residentes nas regiões Centro-oeste, Sudeste e Sul do país. Análi- ses ajustadas pelas variáveis situadas em posição mais distal ao desfecho indicaram desigualdades na prevalência do agravo. A prevalência de dor dentária foi maior nos homens, nos pretos e pardos, nos que não possuem plano de saúde, nos que apresentam maior consumo semanal de refrigerantes e nos fumantes. A prevalência de dor dentária foi $38 \%$ maior nos homens, $73 \%$ maior entre os pretos e $30 \%$ maior entre os pardos comparados com as mulheres e os brancos respectivamente. Dentre os que possuíam plano de saúde observou-se prevalência de dor dentária 39\% menor do que os que não tinham, enquanto que aqueles que apresentaram maior consumo semanal de refrigerantes e eram fumantes as prevalências de dor dentária foram, respectivamente, $31 \%$ e $41 \%$ maiores do que os consumiam menos refrigerante e não fumantes respectivamente (Tabela 2).

A Tabela 3 revela as razões de prevalências da variável contextual - região geográfica - antes e depois do ajuste pelas variáveis individuais. Nota-se que houve uma redução de $26 \%$ na magnitude da razão de prevalência que passou de 1,25 na análise bruta para 1,19 quando ajustada pelas variáveis individuais. Essa observação indica que a variável contextual permaneceu associada à dor dentária independentemente das características individuais.

\section{Discussão}

Este é o primeiro inquérito populacional telefônico realizado no Brasil que investigou a prevalência de dor dentária e características individuais e contextuais associadas. A prevalência de dor dentária, considerando todas as capitais dos estados e Distrito Federal, foi de 15,2\%, resultado muito similar aos $17,7 \%$ e $18 \%$ encontrados entre adultos de Pelotas, Rio Grande do Sul 16 e Lages, Santa Catarina 4 respectivamente, os únicos dois estudos de base populacional realizados no Brasil identificados na literatura que investigaram dor dentária em adultos. Entretanto, o período recordatório de dor dentária entre estes estudos foi diferente, de 6 meses em Pelotas e Lages e um mês para o VIGITEL. Dados de 2002-2003 para o Brasil como um todo, revelaram uma prevalência de dor dentária de $34,8 \%$ entre adultos de 35 a 44 anos 6 , bastante superior ao do presente estudo. Uma das razões para a diferença observada devese ao período recordatório de seis meses no estudo de 2002-2003. Diferenças socioeconômicas entre as populações dos dois inquéritos também podem explicar as diferenças encontradas. Por exemplo, enquanto a escolaridade média entre os brasileiros não ultrapassa os 7 anos, alcança 
Análise multinível de fatores associados à prevalência de dor dentária em adultos e idosos. Sistema de Vigilância de Fatores de Risco e Proteção para Doenças Crônicas por Inquérito Telefônico (VIGITEL), Brasil, 2009.

\begin{tabular}{|c|c|c|c|c|}
\hline Variáveis/Categorias & $\begin{array}{l}\text { RP não } \\
\text { ajustada }\end{array}$ & IC95\% & $\begin{array}{c}\mathrm{RP} \\
\text { ajustada }\end{array}$ & IC95\% \\
\hline \multicolumn{5}{|l|}{ 1ㅇ nível: contexto } \\
\hline \multicolumn{5}{|l|}{ Região geográfica } \\
\hline Sul, Sudeste e Centro-oeste & Referência & & Referência & \\
\hline Norte e Nordeste & 1,25 & $1,10-1,43$ & 1,25 & $1,10-1,43$ \\
\hline \multicolumn{5}{|l|}{ 2o nível: indivíduos } \\
\hline \multicolumn{5}{|l|}{ Características demográficas * } \\
\hline \multicolumn{5}{|l|}{ Faixa etária (anos) } \\
\hline $18-35$ & Referência & & Referência & \\
\hline $36-59$ & 0,68 & $0,60-0,78$ & 0,70 & $0,62-0,79$ \\
\hline$\geq 60$ & 0,43 & $0,36-0,51$ & 0,44 & $0,37-0,52$ \\
\hline \multicolumn{5}{|l|}{ Gênero } \\
\hline Feminino & Referência & & Referência & \\
\hline Masculino & 1,34 & $1,16-1,55$ & 1,38 & $1,20-1,60$ \\
\hline \multicolumn{5}{|l|}{ Raça/Cor da pele } \\
\hline Brancos & Referência & & Referência & \\
\hline Pardos & 1,38 & $1,20-1,57$ & 1,30 & $1,13-1,49$ \\
\hline Pretos & 1,83 & $1,30-2,59$ & 1,73 & $1,23-1,44$ \\
\hline Outros & 1,12 & $0,77-1,80$ & 1,20 & $0,74-1,95$ \\
\hline \multicolumn{5}{|l|}{ Posição socioeconômica ** } \\
\hline \multicolumn{5}{|l|}{ Instrução (anos de estudo) } \\
\hline$\geq 12$ & Referência & & Referência & \\
\hline $9-11$ & 1,02 & $0,94-1,09$ & 1,01 & $0,94-1,09$ \\
\hline $5-8$ & 0,88 & $0,68-1,14$ & 0,86 & $0,69-1,08$ \\
\hline$\leq 4$ & 0,94 & $0,75-1,18$ & 0,92 & $0,76-1,13$ \\
\hline Não informado & 0,88 & $0,48-1,62$ & 0,81 & $0,45-1,43$ \\
\hline \multicolumn{5}{|l|}{ Plano privado de saúde } \\
\hline Não possui & Referência & & Referência & \\
\hline Possui & 0,56 & $0,49-0,64$ & 0,61 & $0,57-0,65$ \\
\hline Não informado & 0,10 & $0,03-0,32$ & 0,11 & $0,02-0,53$ \\
\hline \multicolumn{5}{|l|}{ Comportamento *** } \\
\hline \multicolumn{5}{|c|}{ Consumo de refrigerantes (vezes por semana) } \\
\hline$<3$ & Referência & & Referência & \\
\hline$\geq 3$ & 1,41 & $1,23-1,62$ & 1,31 & $1,09-1,57$ \\
\hline \multicolumn{5}{|l|}{ Hábito de fumar } \\
\hline Não fumantes & Referência & & Referência & \\
\hline Fumantes & 1,49 & $1,20-1,84$ & 1,41 & $1,13-1,76$ \\
\hline
\end{tabular}

IC95\%: intervalo de 95\% de confiança; RP: razão de prevalências.

* Ajustada para região geográfica e características demográficas;

** Ajustada para região geográfica e características demográficas e socioeconômicas;

*** Ajustada para região geográfica e características demográficas, socioeconômicas e comportamentais.

11 entre os participantes do VIGITEL, indicando que a posse de telefone pode ser considerada um indicador social. Outra provável explicação para as diferenças encontradas é a maior proporção de capitais com água fluoretada quando comparada com a proporção da medida entre os municípios brasileiros. A fluoretação das águas reduz a prevalência e extensão da cárie dentária, uma das principais causa de dor dentária. Além disso, o estudo nacional de 2002-2003 investigou 
Redução percentual do efeito de região geográfica na prevalência de dor dentária em adultos e idosos, após ajuste pelos fatores demográficos, socioeconômicos e comportamentais de ordem individual. Sistema de Vigilância de Fatores de Risco e Proteção para Doenças Crônicas por Inquérito Telefônico (VIGITEL), Brasil, 2009.

\begin{tabular}{|c|c|c|}
\hline Fator contextual & RP não ajustada * & IC95\% \\
\hline \multicolumn{3}{|l|}{ Região geográfica } \\
\hline Sul, Sudeste e Centro-oeste & Referência & \\
\hline \multirow[t]{2}{*}{ Norte e Nordeste } & 1,25 & $1,10-1,43$ \\
\hline & RP ajustada ** & IC95\% \\
\hline \multicolumn{3}{|l|}{ Região geográfica } \\
\hline Sul, Sudeste e Centro-oeste & Referência & \\
\hline Norte e Nordeste & 1,19 & $1,13-1,25$ \\
\hline Redução percentual *** & 26,3 & \\
\hline \multicolumn{3}{|c|}{ IC95\%: intervalo de 95\% de confiança; RP: razão de prevalências. } \\
\hline \multicolumn{3}{|c|}{ * Razão de prevalências sem ajuste para fatores demográficos, socioeconômicos e comportamentais; } \\
\hline \multicolumn{3}{|c|}{ ** Razão de prevalências ajustada pelos os fatores demográficos, socioeconômicos e comportamentais; } \\
\hline \multicolumn{3}{|c|}{ *** Redução percentual no efeito de região geográfica após o ajuste por condições demográficas, socioeconômicas e } \\
\hline
\end{tabular}

adultos na faixa etária entre 35 e 44 anos de idade enquanto o de Lages estudou adultos entre 20 e 59 anos, o de Pelotas a partir dos 20 anos e o VIGITEL a partir de 18 anos.

Dentre as limitações do estudo destaca-se o fato de a amostra se restringir àqueles que possuem telefone fixo, com menores coberturas nas regiões Norte e Nordeste, porém o uso de fatores de expansão reduz o viés, buscando aproximar a amostra do estudo da população total residente nas capitais do Brasil, segundo o censo demográfico de 2000 25. Por ser realizado apenas contato telefônico, no caso de recusa na residência ou de recusa do indivíduo entrevistado em participar da pesquisa, não se dispõe de informações a respeito desta pessoa para comparação e análise de possível viés de seleção da amostra. A disparidade entre o número de linhas sorteadas e as efetivamente elegíveis pode ter ocorrido em função de trocas rotineiras de números de telefones ou de prestadoras de serviços, abandono do fixo, ou desatualização dos cadastros telefônicos enviados pelas empresas telefônicas a cada ano para a realização do sorteio. Outra limitação do estudo foi a adoção de duas perguntas em uma mesma (dor de dente e/ou inflamação da gengiva). Neste último caso, a inflamação da gengiva não precisaria estar associada à dor. Esta pergunta já havia sido utilizada na Pesquisa Nacional de Saúde Bucal de 2002-2003 - Projeto SBBrasil 6 - razão de sua utilização no VIGITEL. Recomenda-se a reformulação da pergunta nos futuros inquéritos do VIGITEL, restringindo-a à dor dentária.
Dentre as vantagens do estudo citamos o fato de os inquéritos telefônicos terem uma enorme utilidade devido à sua praticidade, baixo custo e estimativas comparáveis diferentemente de entrevistas face a face 26 .

As variáveis associadas à dor no modelo múltiplo confirmam achados anteriores 4,14,16. Residentes nas regiões Norte e Nordeste, mulheres, os mais jovens, pessoas que se autoclassificaram como de pele parda e preta, os que mais consomem refrigerantes e os fumantes apresentaram maiores prevalências do que os grupos de comparação. Pessoas com plano de saúde, uma variável proxy de condição socioeconômica apresentaram menores prevalências de dor.

As desigualdades regionais nos agravos à saúde são persistentes e também ocorrem com relação à dor dentária. Índice de desenvolvimento humano mais alto foi relatado como associado a menores prevalências de dor em adolescentes de São Paulo 14 mesmo quando controlado pelas variáveis individuais, característica também apresentada no presente estudo. Este achado é importante, pois sugere que o contexto social e econômico exerce influência na dor dentária independente das características individuais dos participantes. As cidades mais pobres apresentam maior prevalência de dor de dente, pois seus cidadãos estão submetidos a um maior risco de doença em virtude de piores padrões alimentares e de higiene bucal, menor acesso e utilização de fluoretos, fatores que influenciam a ocorrência de cárie e doença periodontal, principais causas 
de dor dentária. O acesso e uso de serviços são associados ao contexto socioeconômico de maneira inversa; na década de 1970, Hart 27 formulou a hipótese de que as regiões mais necessitadas de cuidados em saúde careciam de profissionais enquanto o oposto ocorria em regiões mais abastadas, onde as necessidades eram menores. Esta pode ser uma das explicações para a associação encontrada entre os menores IDH e maiores prevalências de dor dentária.

Piores condições socioeconômicas, aferidas por renda, escolaridade e tipo de escola foram associadas à dor dentária em estudos anteriores realizados entre adolescentes 14,28,29,30,31. Neste estudo a escolaridade não foi associada à dor. Entretanto, quando categorizada adotando-se outros pontos de corte, como, por exemplo, menor que 3 anos de estudo, entre 5 a 7,8 a 10 e 11 e mais, manteve-se associada no modelo múltiplo mostrando os menos escolarizados com maiores prevalências de dor. No entanto, a forma adotada neste estudo é a mais frequentemente utilizada na literatura, justificando sua manutenção.

Diferenças de dor dentária segundo cor da pele podem ser atribuídas em parte à confusão residual com variáveis socioeconômicas ou à falta de coleta e análise de variáveis associadas à raça/cor e às condições socioeconômicas, mas, por outro lado podem indicar falta de equidade em relação ao uso e acesso aos serviços de saúde segundo raça. Dados do VIGITEL 200932 revelaram que a falta de acesso a serviços odontológicos foi maior em pardos mesmos após o ajuste por escolaridade, sexo e idade.

A associação entre gênero e dor dentária é controversa. Maior prevalência de dor dentária em mulheres foi verificada no presente estudo e em outros estudos com adolescentes 14,28 e adul- tos 4,16 . Outros pesquisadores, entretanto, não encontraram esta associação 8,29,30,33. Mulheres utilizam e tendem a relatar mais problemas de saúde do que homens, o que pode justificar os resultados 34 .

A associação entre tabagismo e dor de dente confirma os achados de Bastos et al. 16 no Sul do Brasil. A relação entre tabagismo e dor dentária é complexa e pode se dar através de diferentes mecanismos, como via doença periodontal e diminuição do fluxo salivar, o que aumenta o risco de cáries 35 . Outro achado confirmatório deste estudo foi a associação entre alto consumo semanal de refrigerantes e dor dentária. Aproximadamente $40 \%$ dos participantes revelaram consumir refrigerantes, comumente açucarados, o que contribui para o desenvolvimento de lesões de cáries e consequentemente de dor. Níveis elevados de consumo de tabaco e refrigerantes em escala populacional constituem-se em fatores de risco comuns para doenças e agravos crônicos à saúde geral e bucal indicando a necessidade de estratégias de risco comuns dirigidas para sua prevenção 36 .

A prevalência de dor dentária de $15 \%$, o conhecimento do impacto negativo que ela exerce nas atividades da vida diária, a confirmação das persistentes desigualdades regionais e segundo características individuais, sua associação com exposições nocivas à saúde geral, como tabaco e consumo de refrigerantes, caracterizam a dor dentária como um problema de Saúde Pública. A inserção periódica de questões relacionadas à dor dentária e outros problemas bucais como blocos rotativos no VIGITEL permitirá o monitoramento deste e outros agravos ao longo do tempo, subsidiando políticas para o seu enfrentamento e contribuindo para a consolidação da vigilância em saúde bucal. 


\section{Resumo}

O objetivo deste estudo foi conhecer a prevalência de dor dentária e fatores associados em adultos e idosos residentes nas capitais brasileiras usando os dados do Sistema de Vigilância de Fatores de Risco e Proteção para Doenças Crônicas por Inquérito Telefônico (VIGITEL), de 2009 ( $n=54.367)$. Dor dentária foi a variável dependente. Macrorregião, idade, sexo, raça, escolaridade, posse de plano de saúde, tabagismo e consumo de refrigerantes foram as variáveis exploratórias. Foram realizadas regressões de Poisson multinível. A prevalência de dor dentária foi de 15,2\%; Macapá e São Luís apresentaram prevalências maiores que 20\% enquanto em todas as capitais do Sul e Sudeste, em Cuiabá, Campo Grande, Maceió, Recife e Natal foram encontradas prevalências menores que 15\%. Residentes no Norte e Nordeste, mulheres, pretos e pardos, aqueles que não possuem plano de saúde, tabagistas e consumidores de refrigerantes apresentaram as maiores prevalências de dor dentária. A dor dentária é um problema de saúde pública que deve ser monitorado pelos sistemas de vigilância em saúde.

Odontalgia; Inquéritos Epidemiológicos; Inquéritos de Saúde Bucal; Saúde Bucal

\section{Referências}

1. Locker D, Grushka M. The impact of dental and facial pain. J Dent Res 1987; 66:1414-7.

2. Jamieson LM, Roberts-Thomson KF, Sayers SM. Risk indicators for severe impaired oral health among indigenous Australian young adults. BMC Oral Health 2010; 10:1.

3. Riley 3rd JL, Gilbert GH. Orofacial pain symptoms: an interaction between age and sex. Pain 2001; 90:245-56.

4. Kuhnen M, Peres MA, Masiero AV, Peres KG. Toothache and associated factors in Brazilian adults: a cross-sectional population-based study. BMC Oral Health 2009; (9):7.

5. MacFarlane TV, Blinkhorn AS, Davies RM, Kincey J, Worthington HV. Oro-facial pain in the community: prevalence and associated impact. Community Dent Oral Epidemiol 2002; 30:52-60.

6. Coordenação Nacional de Saúde Bucal, Departamento de Atenção Básica, Secretaria de Atenção à Saúde, Ministério da Saúde. Projeto SB Brasil 2003. Condições de saúde bucal da população brasileira 2002-2003 - resultados principais. Brasília: Ministério da Saúde; 2004.

\section{Colaboradores}

M. A. Peres concebeu o estudo, participou das análises e interpretação dos dados e redigiu o manuscrito. B. P. M. Iser revisou a literatura, interpretou os dados e redigiu o manuscrito. K. G. Peres e D. C. Malta interpretaram os dados e redigiram o manuscrito. J. L. F. Antunes realizou as análises, interpretou os dados e redigiu o manuscrito.
7. Lisboa IC, Abegg C. Hábitos de higiene bucal e uso de serviços odontológicos por adolescentes e adultos do Município de Canoas, Estado do Rio Grande do Sul, Brasil. Epidemiol Serv Saúde 2006; 15:29-39.

8. Lacerda JT, Simionato EM, Peres KG, Peres MA, Traebert J, Marcenes W. Dental pain as the reason for visiting a dentist in a Brazilian adult population. Rev Saúde Pública 2004; 38:453-8.

9. Alexandre GC, Nadanovsky P, Lopes CS, Faerstein E. Prevalência e fatores associados à ocorrência da dor de dente que impediu a realização de tarefas habituais em uma população de funcionários públicos no Rio de Janeiro, Brasil. Cad Saúde Pública 2006; 22:1073-8.

10. Gilbert GH, Duncan RP, Heft MW, Dolan TA, Vogel WB. Oral disadvantage among dentate adults. Community Dent Oral Epidemiol 1997; 25:301-13.

11. Honkala E, Honkala S, Rimpela A, Rimpela M. The trend and risk factors of perceived toothache among Finnish adolescents from 1977 to 1997. J Dent Res 2001; 80:1823-7. 
12. Vargas CM, Macek MD, Marcus SE. Sociodemographic correlates of tooth pain among adults: United States, 1989. Pain 2000; 85:87-92.

13. Slade GD. Epidemiology of dental pain and dental caries among children and adolescents. Community Dent Health 2001; 18:219-27.

14. Peres MA, Peres KG, Frias AC, Antunes JL. Contextual and individual assessment of dental pain period prevalence in adolescents: a multilevel approach. BMC Oral Health 2010; 10:20.

15. Lahti S, Sipila K, Taanila A, Laitinen J. Oral pain and associated factors among adolescents in northern Finland. Int J Circumpolar Health 2008; 67:245-53.

16. Bastos JL, Gigante DP, Peres KG. Toothache prevalence and associated factors: a population based study in southern Brazil. Oral Dis 2008; 14:320-6.

17. Bastos JL, Gigante DP, Peres KG, Nedel FB. Social determinants of odontalgia in epidemiological studies: theoretical review and proposed conceptual model. Ciênc Saúde Coletiva 2007; 12:1611-21.

18. Instituto Brasileiro de Geografia e Estatística. Pesquisa Nacional de Saúde do Escolar (PeNSE) 2009. Rio de Janeiro: Instituto Brasileiro de Geografia e Estatística; 2009.

19. Ministério da Saúde. Planejamento da Pesquisa Nacional de Saúde (PNS). http://portal.saude.gov. $\mathrm{br} /$ portal/saude/visualizar_texto.cfm?idtxt=35038 (acessado em 20/Out/2010).

20. Departamento de Análise de Situação de Saúde, Secretaria de Vigilância em Saúde, Ministério da Saúde. Vigilância de fatores de risco e proteção para doenças crônicas por inquérito telefônico, Vigitel 2009. Brasília: Ministério da Saúde; 2010.

21. Moura EC, Neto OL, Malta DC, Moura L, Silva NN, Bernal R, et al. Vigilância de Fatores de Risco para Doenças Crônicas por Inquérito Telefônico nas capitais dos 26 estados brasileiros e no Distrito Federal (2006). Rev Bras Epidemiol 2008; 11 Suppl 1: 20-37.

22. Programa das Nações Unidas para o Desenvolvimento/Instituto de Pesquisa Econômica Aplicada/ Fundação João Pinheiro/Instituto Brasileiro de Geografia e Estatística. Atlas do desenvolvimento humano no Brasil. Brasília: Programa das Nações Unidas para o Desenvolvimento; 2003.

23. Snijders TAB, Bosker RJ. Multilevel analysis. An introduction to basic and advanced multilevel modelling. London: Sage Publications; 1999.

24. Victora CG, Huttly SR, Fuchs SC, Olinto MT. The role of conceptual frameworks in epidemiological analysis: a hierarchical approach. Int J Epidemiol 1997; 26:224-7.
25. Bernal R, Silva NN. Home landline telephone coverage and potential bias in epidemiological surveys. Rev Saúde Pública 2009; 43:421-6.

26. Nelson DE, Powell-Griner E, Town M, Kovar MG. A comparison of national estimates from the National Health Interview Survey and the Behavioral Risk Factor Surveillance System. Am J Public Health 2003; 93:1335-41.

27. Hart JT. The inverse care law. Lancet 1971; 1:405-12.

28. Bastos JL, Peres MA, Peres KG, Araujo CL, Menezes AM. Toothache prevalence and associated factors: a life course study from birth to age $12 \mathrm{yr}$. Eur J Oral Sci 2008; 116:458-66.

29. Nomura LH, Bastos JLD, Peres MA. Dental pain prevalence and association with dental caries and socioeconomic status in schoolchildren, Southern Brazil, 2002. Braz Oral Res 2004; 18:134-40.

30. Goes PS, Watt R, Hardy RG, Sheiham A. The prevalence and severity of dental pain in 14-15 year old Brazilian schoolchildren. Community Dent Health 2007; 24:217-24.

31. Hack-Comunello SM, Michel-Crosato E, Biazevic MGH, Crosato E. Dor dental e condição sócio-econômica: um estudo censitário em escolares. Pesq Bras Odontoped Clin Integr 2008; 8:63-7.

32. Peres MA, Iser BPM, Boing AF, Yokota RTC, Malta DC, Peres KG. Desigualdades no acesso e na utilização de serviços odontológicos no Brasil: análise do Sistema de Vigilância de Fatores de Risco e Proteção para Doenças Crônicas por Inquérito Telefônico (VIGITEL 2009). Cad Saúde Pública 2012; 28 Suppl:S90-S100.

33. Barretto EP, Ferreira EF, Pordeus IA. Determinant factors of toothache in 8- and 9-year-old schoolchildren, Belo Horizonte, MG, Brazil. Braz Oral Res 2009; 23:124-30.

34. Lima-Costa MF, Peixoto SV, Firmo JO. Validity of self-reported hypertension and its determinants (the Bambui study). Rev Saúde Pública 2004; 38:637-42.

35. Riley 3rd JL, Tomar SL, Gilbert GH. Smoking and smokeless tobacco: increased risk for oral pain. J Pain 2004; 5:218-25.

36. Sheiham A, Watt RG. The common risk factor approach: a rational basis for promoting oral health. Community Dent Oral Epidemiol 2000; 28:399-406.

Recebido em 22/Dez/2010

Versão final reapresentada em 18/Jul/2011

Aprovado em 25/Jul/2011 